\title{
On Some Diagram Assertions in Preabelian and $P$-Semi-Abelian Categories
}

\begin{abstract}
Ya. A. Kopylov
Yaroslav A. Kopylov, https://orcid.org/0000-0002-0343-4424, Sobolev Institute of Mathematics, 4 Ac. Koptyuga Ave., Novosibirsk 630090, Russia, yakop@math.nsc.ru

As is well known, many important additive categories in functional analysis and algebra are not abelian. Many classical diagram assertions valid in abelian categories fail in more general additive categories without additional assumptions concerning the properties of the morphisms of the diagrams under consideration. This in particular applies to the so-called Snake Lemma, or the KerCoker-sequence. We obtain a theorem about a diagram generalizing the classical situation of the Snake Lemma in the context of categories semi-abelian in the sense of Palamodov. It is also known that, already in $P$-semi-abelian categories, not all kernels (respectively, cokernels) are semi-stable, that is, stable under pushouts (respectively, pullbacks). We prove a proposition showing how non-semi-stable kernels and cokernels can arise in general preabelian categories.
\end{abstract}

Keywords: $P$-semi-abelian category, strict morphism, semi-stable kernels and cokernels, Snake Lemma, Ker-sequence, Coker-sequence.

Received: 15.12.2019 / Accepted: 23.03.2020 / Published: 30.11.2020

This is an open access article distributed under the terms of Creative Commons Attribution License (CC-BY 4.0)

DOI: https://doi.org/10.18500/1816-9791-2020-20-4-434-443

\section{INTRODUCTION}

As is well known, many classical diagram assertions valid in abelian categories fail in more general additive and nonadditive categories without additional assumptions concerning the properties of the morphisms of the diagrams under consideration. This in particular applies to the so-called Snake Lemma, or the Ker-Coker-sequence (see, for example, [1,2] or [3]). It is natural to expect that possible generalizations of the Snake Lemma in the non-abelian setting would also require additional conditions on the morphisms of the diagram under consideration.

In the present article, we consider a diagram of the form

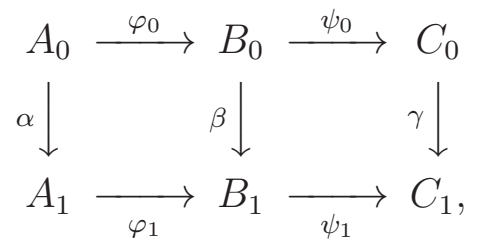

where $\psi_{0} \varphi_{0}=0$ and $\psi_{1} \varphi_{1}=0$, in $P$-semi-abelian categories, a class of additive categories with kernels and cokernels which appeared under different names in the 1960s in the works of Romanian mathematicians (see [4]) and were studied in more detail by D. A. Raikov (under the name of "preabelian") in [5] and V. P. Palamodov in [6].

In [7, Corollary A2], Nomura proved an assertion about the exactness of the Ker- and Coker-sequences corresponding to a diagram of the form (1) in a Puppe exact category, that is, informally speaking, in an "abelian category without additivity". In [2], we proved a version of Nomura's assertion for quasi-abelian categories. It turned out that 
an analog of this assertion also holds for the larger class of $P$-semi-abelian categories. This is the main result of the present article.

The article is organized as follows.

In Section 1., we give the necessary definitions and recall some basic facts. In Section 2., we discuss one way for obtaining non-semi-stable kernels and cokernels in a preabelian category. In Section 4., we prove the above mentioned main result on the exactness of the Ker- and Coker-sequences (Theorem 1).

\section{PREABELIAN AND $P$-SEMI-ABELIAN CATEGORIES}

We consider preabelian categories, i.e., additive categories satisfying the following axiom.

Axiom 1. Each morphism has a kernel and a cokernel.

We denote an arbitrary kernel (cokernel) of $\alpha$ by ker $\alpha$ (coker $\alpha$ ) and the corresponding object by $\operatorname{Ker} \alpha(\operatorname{Coker} \alpha)$; the equality $a=\operatorname{ker} b(a=\operatorname{coker} b)$ means that $a$ is a kernel of $b$ ( $a$ is a cokernel of $b)$.

In a preabelian category, every morphism $\alpha$ admits a canonical decomposition $\alpha=(\operatorname{im} \alpha) \bar{\alpha}(\operatorname{coim} \alpha)$, where $\operatorname{im} \alpha=\operatorname{ker} \operatorname{coker} \alpha, \operatorname{coim} \alpha=$ coker $\operatorname{ker} \alpha$. A morphism $\alpha$ is called strict if $\bar{\alpha}$ is an isomorphism.

We write $\alpha \mid \beta$ if $\alpha=\operatorname{ker} \beta$ and $\beta=\operatorname{coker} \alpha$.

Lemma 1. [4,8-10] The following assertions hold in a preabelian category:

(i) a strict monomorphism is the same as a kernel; a strict epimorphism is the same as a cokernel;

(ii) $\alpha$ is a kernel $\Longleftrightarrow \alpha=\operatorname{im} \alpha, \alpha$ is a cokernel $\Longleftrightarrow \alpha=\operatorname{coim} \alpha$;

(iii) a morphism $\alpha$ is strict if and only if it is representable in the form $\alpha=\alpha_{1} \alpha_{0}$ with $\alpha_{0}$ a cokernel and $\alpha_{1}$ a kernel; in every such representation, $\alpha_{0}=\operatorname{coim} \alpha$ and $\alpha_{1}=\operatorname{im} \alpha$;

(iv) the relations $\operatorname{ker} \alpha=\operatorname{ker} \operatorname{coim} \alpha$ and $\operatorname{coker} \alpha=$ coker im $\alpha$ hold for every morphism $\alpha$.

A preabelian category is abelian if and only if $\bar{\alpha}$ is an isomorphism for every $\alpha$, that is, if and only if every morphism is strict.

We call a sequence $\ldots \stackrel{a}{\rightarrow} B \stackrel{b}{\rightarrow} \ldots$ in an additive category semi-exact at the term $B$ if $b a=0$. A sequence $\ldots \stackrel{a}{\rightarrow} B \stackrel{b}{\rightarrow} \ldots$ in a preabelian category is said to be exact at the term $B$ if $\operatorname{im} a=\operatorname{ker} b$. Lemma 1(iv), which is Lemma 1 of [10], implies that the sequence is exact at the term $B$ if and only if $\operatorname{coker} a=\operatorname{coim} b$.

A preabelian category is called P-semi-abelian or semi-abelian (in the sense of Palamodov) [6,11] if it satisfies

Axiom 2. For every morphism $\alpha, \bar{\alpha}$ is a bimorphism, that is, a monomorphism and an epimorphism.

If the morphism $\bar{\alpha}$ is a monomorphism (an epimorphism) for every $\alpha$ then, following Rump [11, p. 167], we call the preabelian category left semi-abelian (right semi-abelian).

Note that a preabelian category is right (left) semi-abelian if and only if the composition of any two kernels (respectively, cokernels) in it is again a kernel (respectively, a cokernel), which is equivalent to the statement that if $g f$ is a kernel then $f$ is a kernel (if $g f$ is a cokernel then $g$ is a cokernel). For a detailed characterization of $P$-semiabelianity, the reader is referred to [12]. 
A preabelian category $\mathscr{A}$ is called left quasi-abelian (or left almost abelian, see [11]) if it satisfies

Axiom 3. If a commutative square

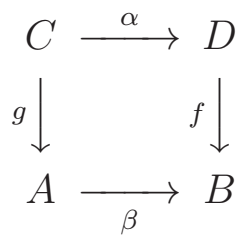

is a pullback then $f$ is a cokernel $\Longrightarrow g$ is a cokernel.

Dually, a preabelian category $\mathscr{A}$ is called right quasi-abelian (or right almost abelian [11]) if it satisfies

Axiom $3^{*}$. If (2) is a pushout then $g$ is a kernel $\Longrightarrow f$ is a kernel.

A left and right quasi-abelian category is referred to as quasi-abelian [13] (semiabelian in the sense of Raikov [5], or almost abelian [11]).

As is well-known $[5,9,11,13]$, every quasi-abelian category is $P$-semi-abelian. Kuz'minov and Cherevikin [9, Theorem 2] and later Rump [11, Proposition 3] noticed that a $P$-semi-abelian category is quasi-abelian if and only if it is left or right quasiabelian. In 2006, Bonet and Dierolf [14] constructed an example of a pullback violating Axiom 3 in the category Bor of bornological locally convex spaces, thus proving that it is not quasi-abelian. Later Rump [15] gave an algebraic example of a $P$-semi-abelian but not quasi-abelian category. In [16], he carried out a thorough study of $P$-semi-abelian subcategories of quasi-abelian categories and proved that Bor and the category Bar of barreled locally convex spaces are $P$-semi-abelian but not quasi-abelian. Later in [17] Wengenroth explained that the non-semi-stability of cokernels in Bor is not rare.

\section{SEMI-STABLE KERNELS AND COKERNELS IN A PREABELIAN CATEGORY}

If, for a cokernel $f$ in a preabelian category, in every pullback (2), $g$ is a cokernel (for a kernel $g$ in a preabelian category, in every pushout (2), $f$ is a kernel) then $f$ is called a semi-stable cokernel ( $g$ is called a semi-stable kernel).

We recall some basic properties of semi-stable kernels and cokernels (following from [18, Propositions 5.11 and 5.12]).

Lemma 2. The following hold in a preabelian category:

(i) if $g f$ is a semi-stable kernel then so is $f$, if $g f$ is a semi-stable cokernel then so is $g$;

(ii) if $f$ and $g$ are semi-stable kernels and $g f$ is defined then $g f$ is a semi-stable kernel; if $f$ and $g$ are semi-stable cokernels and $g f$ is defined then $g f$ is a semi-stable cokernel;

(iii) a pushout of a semi-stable kernel is a semi-stable kernel; a pullback of a semi-stable cokernel is a semi-stable cokernel.

The following Lemma is due to Kuz'minov and Cherevikin [9, Lemma 2].

Lemma 3. Let

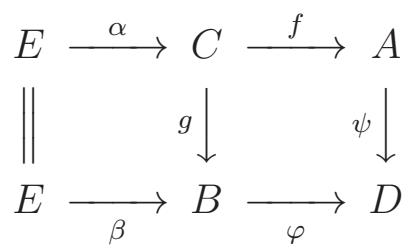


be a commutative diagram in a preabelian category. Assume that $f \alpha=0, f$ is an epimorphism, and $\varphi=\operatorname{coker} \beta$. Then the $\varphi g=\psi f$ is a pushout.

The dual assertion also holds.

The idea of the following assertion, allowing to construct examples of non-semistable kernels and cokernels, comes from the proof of [9, Theorem 1(3)].

Proposition 1. Let $\alpha$ be a morphism in a preabelian category for which $\bar{\alpha}$ is not an epimorphism. Then im $\alpha$ is a non-semi-stable kernel.

By duality, if a morphism $\alpha$ is such that $\bar{\alpha}$ is not a monomorphism then $\operatorname{coim} \alpha$ is a non-semi-stable cokernel.

Proof. Let $\alpha: A \rightarrow B$ be a morphism such that $\bar{\alpha}$ is not epic and $\beta$ is the morphism of the cokernels of the rows in the commutative square

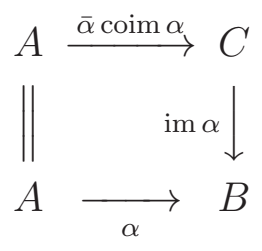

Then the commutative diagram

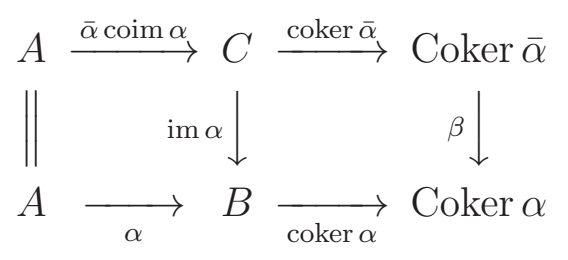

satisfies the hypothesis of Lemma 3. Thus, $\beta \operatorname{coker} \bar{\alpha}=(\operatorname{coker} \alpha) \operatorname{im} \alpha(=0)$ is a pushout. Since coker $\bar{\alpha}$ is epic, the equality $\beta$ coker $\bar{\alpha}=0$ implies that $\beta=0$. In particular, since coker $\bar{\alpha} \neq 0$, we infer that $\beta$ is not a monomorphism and, thus, im $\alpha$ is a non-semi-stable kernel.

The second assertion of the lemma is obtained from the first by duality.

\section{THE LEFT AND RIGHT HOMOLOGY OBJECTS}

Suppose first that the ambient category is preabelian.

Given a sequence of the form

$$
A \stackrel{\varphi}{\rightarrow} B \stackrel{\psi}{\rightarrow} C
$$

such that $\psi \varphi=0$, there are a natural morphism $\sigma: A \rightarrow \operatorname{Ker} \psi$ such that $\varphi=(\operatorname{ker} \psi) \sigma$ and a natural morphism $\tau$ : Coker $\varphi \rightarrow C$ such that $\psi=\tau \operatorname{coker} \varphi$.

Definition 1. Call $H_{-}(B)=H_{-}(B, \varphi, \psi)=$ Coker $\sigma$ and $H_{+}(B)=H_{+}(B, \varphi, \psi)=$ $=\operatorname{Ker} \tau$ the left and right homology objects of (3) at the term $B$.

It is classical that these two notions coincide for abelian categories (see, for example, [19]). This remains valid for quasi-abelian categories [20] and even in the nonadditive setting of homological categories in the sense of Grandis [3].

If the ambient category is $P$-semi-abelian then there is an equivalent description of the left and right homology objects. Consider the natural morphisms $r: \operatorname{Im} \varphi \rightarrow \operatorname{Ker} \psi$ 
and $r^{\prime}: \operatorname{Coker} \varphi \rightarrow \operatorname{Coim} \psi$. Then $\operatorname{coker} r=\operatorname{coker} \sigma$ and $\operatorname{ker} r^{\prime}=\operatorname{ker} \tau$, and hence $H_{-}(B, \varphi, \psi)=$ Coker $r$ and $H_{+}(B, \varphi, \psi)=\operatorname{Ker} r^{\prime}$.

As was shown in [20], in a preabelian category, there is a unique morphism $m: H_{-}(B) \rightarrow H_{+}(B)$ such that

$$
(\operatorname{ker} \tau) m \operatorname{coker} \sigma=(\operatorname{coker} \varphi)(\operatorname{ker} \psi) \text {. }
$$

The following assertion holds ([21, Lemma 7], [22, Proposition 1]).

Lemma 4. ( $i)$ Let the ambient category be P-semi-abelian. The morphism $m: H_{-}(B) \rightarrow H_{+}(B)$ is a bimorphism. If $\operatorname{ker} \psi$ is a semi-stable kernel or coker $\varphi$ is a semi-stable cokernel then $m$ is an isomorphism.

(ii) Let the ambient category be preabelian. If ker $\psi$ is a semi-stable kernel then $m$ is a semi-stable kernel and if coker $\varphi$ is a semi-stable cokernel then $m$ is a semi-stable cokernel. Thus, if both conditions are fulfilled then $m$ is an isomorphism.

Examples of situations when the left and right homology objects do not coincide can be obtained from the following observation [22, Lemma 4]:

Let

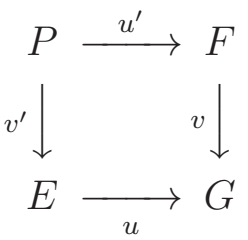

be a pullback in a P-semi-abelian category such that $v$ is a kernel, $u$ is a cokernel, and $u^{\prime}$ is not a cokernel. Let $H_{-}(E)$ and $H_{+}(E)$ be the left and right homology objects of the sequence

$$
K \stackrel{\operatorname{ker} u}{\longrightarrow} E \stackrel{\text { coker } v^{\prime}}{\longrightarrow} L
$$

at the term $E$. Then the canonical morphism $m: H_{-}(E) \rightarrow H_{+}(E)$ is not an isomorphism.

As was shown by Wengenroth (see [17]), such pullbacks are not unusual, for example, in the $P$-semi-abelian category of bornological locally convex spaces and arise when non- $\alpha$-regular inductive limits in the sense of Makarov [23] are considered.

\section{A GENERALIZATION OF THE SNAKE LEMMA}

Consider a diagram of the form (1) in a $P$-semi-abelian category.

As in the case of the classical diagram of the Snake Lemma, diagram (1) gives rise to a Ker-sequence

$$
\operatorname{Ker} \alpha \stackrel{\varepsilon}{\rightarrow} \operatorname{Ker} \beta \stackrel{\zeta}{\rightarrow} \operatorname{Ker} \gamma
$$

with $\zeta \varepsilon=0$ and a Coker-sequence

$$
\text { Coker } \alpha \stackrel{\tau}{\rightarrow} \text { Coker } \beta \stackrel{\theta}{\rightarrow} \text { Coker } \gamma
$$

with $\theta \tau=0$.

For diagram (1), we have a commutative diagram of natural morphisms

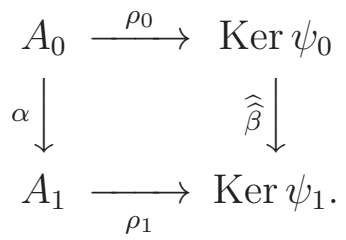


Here $\widehat{\widehat{\beta}}:$ Ker $\psi_{0} \rightarrow \operatorname{Ker} \psi_{1}$ is the natural morphism of the kernels of the rows of the square

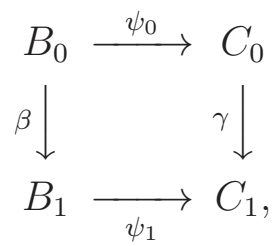

and $\rho_{0}$ and $\rho_{1}$ are uniquely defined by $\varphi_{0}=\left(\operatorname{ker} \psi_{0}\right) \rho_{0}$ and $\varphi_{1}=\left(\operatorname{ker} \psi_{1}\right) \rho_{1}$. We thus have a natural morphism $\chi_{-}: H_{-}\left(B_{0}\right) \rightarrow H_{-}\left(B_{1}\right)$ of the cokernels of the rows in (7) such that

$$
\chi_{-} \operatorname{coker} \rho_{0}=\left(\operatorname{coker} \rho_{1}\right) \widehat{\widehat{\beta}} \text {. }
$$

In the dual manner, we have a commutative diagram of natural morphisms

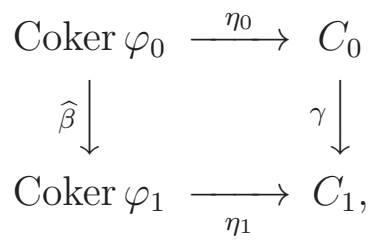

where $\widehat{\beta}$ is the morphism of the cokernels of the rows of the square

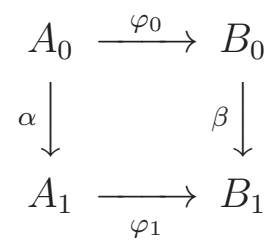

and $\eta_{0}$ and $\eta_{1}$ are uniquely defined by $\psi_{0}=\eta_{0}$ coker $\varphi_{0}$ and $\psi_{1}=\eta_{1}$ coker $\varphi_{1}$. This gives a natural morphism $\chi_{+}: H_{+}\left(B_{0}\right) \rightarrow H_{+}\left(B_{1}\right)$ of the kernels of the rows in (8) such that

$$
\widehat{\beta} \operatorname{ker} \eta_{0}=\left(\operatorname{ker} \eta_{1}\right) \chi_{+} \text {. }
$$

In [2], we proved the following assertion (Lemma 10):

Suppose in (1) that $\varphi_{0}=\operatorname{ker} \psi_{0}\left(\psi_{1}=\right.$ coker $\left.\psi_{1}\right)$. Then $\varepsilon=\operatorname{ker} \zeta$ (respectively, $\theta=\operatorname{coker} \tau)$.

We will prove the following generalization of this assertion, which is a $P$-semiabelian version of [7, Corollary A2] and [2, Theorem 4].

Theorem 1. The following hold:

(1) if, in a diagram of the form (1) in a P-semi-abelian category, the morphism $\varphi_{0}$ is strict and $\varphi_{1}$ and $\chi_{-}: H_{-}\left(B_{0}\right) \rightarrow H_{-}\left(B_{1}\right)$ are monomorphisms then sequence (5) is exact at the term $\operatorname{Ker} \beta$;

(2) if, in a diagram of the form (1) in a P-semi-abelian category, the morphism $\psi_{1}$ is strict and $\psi_{0}$ and $\chi_{+}: H_{+}\left(B_{0}\right) \rightarrow H_{+}\left(B_{1}\right)$ are epimorphisms then sequence (6) is exact at the term Coker $\beta$.

Proof. 1. Take a morphism $x: X \rightarrow \operatorname{Ker} \beta$ with $\zeta x=0$. Show that $x=(\operatorname{im} \varepsilon) \tilde{x}$ for some unique $\tilde{x}$. We may assume without loss of generality that $x=\operatorname{im} x$. 
Since $0=(\operatorname{ker} \gamma) \zeta x=\psi_{0}(\operatorname{ker} \beta) x$, there is a morphism $z: X \rightarrow \operatorname{Ker} \psi_{0}$ such that $(\operatorname{ker} \beta) x=\left(\operatorname{ker} \psi_{0}\right) z$. Further, $\left(\operatorname{ker} \psi_{1}\right) \widehat{\widehat{\beta}} z=\beta\left(\operatorname{ker} \psi_{0}\right) z=\beta(\operatorname{ker} \beta) x=0$ and $\operatorname{ker} \psi_{1}$ is a monomophism; therefore, $\widehat{\widehat{\beta}} z=0$. Let $r_{0}$ be the natural morphism $\operatorname{Im} \varphi_{0} \rightarrow \operatorname{Ker} \psi_{0}$ such that $\operatorname{im} \varphi_{0}=\left(\operatorname{ker} \psi_{0}\right) r_{0}$. Since $\chi\left(\operatorname{coker} \rho_{0}\right) z=\left(\operatorname{coker} \rho_{1}\right) \widehat{\widehat{\beta}} z=0$ and $\chi$ is a monomorphism, we get $\left(\operatorname{coker} \rho_{0}\right) z=0$. Note that $r_{0}=\operatorname{ker}\left(\operatorname{coker} \rho_{0}\right)=\operatorname{im} \rho_{0}$. Hence, $z=r_{0} \mu$ for some $\mu$.

Let $r_{1}$ be the natural morphism $\operatorname{Im} \varphi_{1} \rightarrow \operatorname{Ker} \psi_{1}$ such that $\operatorname{im} \varphi_{1}=\left(\operatorname{ker} \psi_{1}\right) r_{1}$. As was observed in Section 3., coker $\rho_{0}=$ coker $r_{0}$ and coker $\rho_{1}=$ coker $r_{1}$. Let $s: \operatorname{Im} \varphi_{0} \rightarrow \operatorname{Im} \varphi_{1}$ be the natural morphism of the kernels of the rows of the square

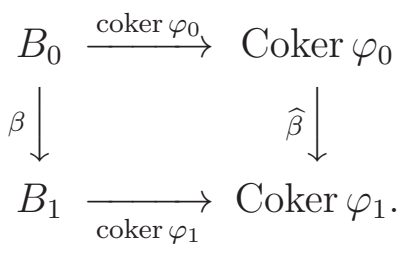

We have the commutative diagram

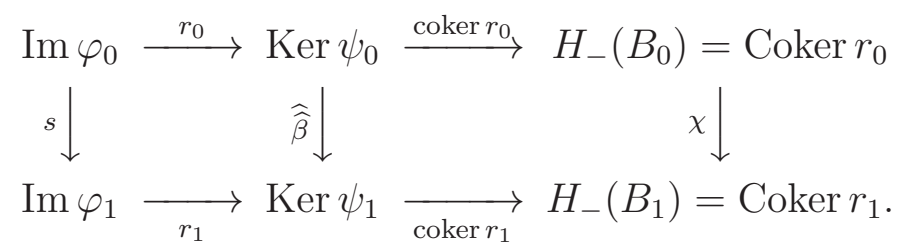

We infer

$$
r_{1} s \mu=\widehat{\widehat{\beta}} r_{0} \mu=\widehat{\widehat{\beta}} \mu=0 .
$$

Since $r_{1}$ is a monomorphism, this gives $s \mu=0$.

Represent $\varphi_{0}$ in the form $\varphi_{0}=\left(\operatorname{im} \varphi_{0}\right) \varphi_{0}^{\prime}$. Since $\varphi_{0}$ is strict, $\varphi_{0}^{\prime}$ is a cokernel. Consider the pullback

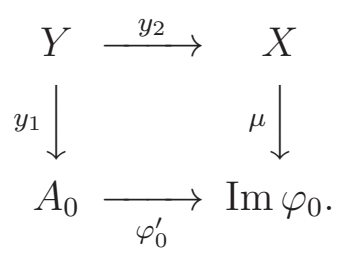

Then $y_{2}$ is an epimorphism. Observe that $\beta \operatorname{im} \varphi_{0}=\left(\operatorname{im} \varphi_{1}\right) s$. We infer

$$
\varphi_{1} \alpha y_{1}=\beta \varphi_{0} y_{1}=\beta\left(\operatorname{im} \varphi_{0}\right) \varphi_{0}^{\prime} y_{1}=\beta\left(\operatorname{im} \varphi_{0}\right) \mu y_{2}=\left(\operatorname{im} \varphi_{1}\right) s \mu y_{2}=0 .
$$

But $\varphi_{1}$ is a monomorphism; therefore, $\alpha y_{1}=0$. Hence, there exists a morphism $y: Y \rightarrow \operatorname{Ker} \alpha$ with the property $y_{1}=(\operatorname{ker} \alpha) y$. Then

$$
\begin{gathered}
(\operatorname{ker} \beta) x y_{2}=\left(\operatorname{ker} \psi_{0}\right) z y_{2}=\left(\operatorname{ker} \psi_{0}\right) r_{0} \mu y_{2}= \\
=\left(\operatorname{im} \varphi_{0}\right) \mu y_{2}=\left(\operatorname{im} \varphi_{0}\right) \varphi_{0}^{\prime} y_{1}=\varphi_{0} y_{1}=\varphi_{0}(\operatorname{ker} \alpha) y=(\operatorname{ker} \beta) \varepsilon y .
\end{gathered}
$$

Since $\operatorname{ker} \beta$ is a monomorphism, this yields

$$
x y_{2}=\varepsilon y .
$$

Let $\varepsilon=(\operatorname{im} \varepsilon) \varepsilon^{\prime}$. In (9), $x$ is a kernel, $y_{2}$ is an epimorphism; therefore, $x=\operatorname{im}\left(x y_{2}\right)=$ $=(\operatorname{im} \varepsilon)\left(\operatorname{im}\left(\varepsilon^{\prime} y\right)\right)$. We can take $\tilde{x}=\operatorname{im}\left(\varepsilon^{\prime} y\right)$. The condition $x=(\operatorname{im} \varepsilon) \tilde{x}$ defines $\tilde{x}$ uniquely because $\mathrm{im} \varepsilon$ is a monomorphism. 
Assertion 1 of the theorem is proved. Assertion 2 results from it by duality.

The theorem is proved.

Let us formulate explicitly what Theorem 1 means for the categories Bor and Bar of bornological and barreled locally convex spaces respectively.

We say that a sequence $A \stackrel{\varphi}{\rightarrow} B \stackrel{\psi}{\rightarrow} C$ in either of these categories such that $\psi \varphi=0$ is approximately exact at the term $B$ whenever the closure of the range of the operator $\varphi$ coincides with the kernel of $\psi$. It is not hard to see that our categorical exactness is in fact approximate exactness in this sense. Moreover, a continuous linear operator between bornological or barreled spaces is strict if and only if it has closed range and is an open mapping onto its range.

Corollary. Consider a commutative diagram of the form (1) constituted by bornological or barreled locally convex spaces and continuous linear operators. The following hold:

(1) if in (1) the operator $\varphi_{0}$ has closed range and is open onto its range $\varphi_{1}$ and $\chi_{-}: H_{-}\left(B_{0}\right) \rightarrow H_{-}\left(B_{1}\right)$ are injective then the corresponding left sequence (5) is approximately exact at the term $\operatorname{Ker} \beta$;

(2) if in (1) the operator $\psi_{1}$ has closed range and is open onto its range and $\psi_{0}$ and $\chi_{+}: H_{+}\left(B_{0}\right) \rightarrow H_{+}\left(B_{1}\right)$ have dense range then the corresponding sequence (6) is approximately exact at the term Coker $\beta$.

Acknowledgements: The author is indebted to the referee for valuable remarks which substantially improved the exposition. The work was carried out in the framework of the State Contract of the Sobolev Institute of Mathematics (project No. 0314-20190006).

\section{References}

1. Kopylov Ya. A., Kuz'minov V. I. On the Ker-Coker-sequence in a semiabelian category. Siberian Math. J., 2000, vol. 41, no. 3, pp. 509-517. DOI: https://doi.org/10.1007/BF02674106

2. Kopylov Ya. A., Kuz'minov V. I. The Ker-Coker-sequence and its generalization in some classes of additive categories. Siberian Math. J., 2009, vol. 50, no. 1, pp. 86-95. DOI: https://doi.org/10.1007/s11202-009-0010-y

3. Grandis M. On the categorical foundations of homological and homotopical algebra. Cah. Topol. Géom. Différ. Catég., 1992, vol. 33, no. 2, pp. 135-175.

4. Bucur I., Deleanu A. Introduction to the Theory of Categories and Functors. London, New York, Sydney, Interscience Publ., John Wiley \& Sons, Ltd., 1968. 224 p.

5. Raikov D. A. Semiabelian categories. Soviet Math. Dokl., 1969, vol. 10, pp. 1242-1245.

6. Palamodov V. P. Homological methods in the theory of locally convex spaces. Russ. Math. Surv., 1971, vol. 26, iss. 1, pp. 1-64. DOI: http://dx.doi.org/10.1070/RM1971v026n01ABEH003815

7. Nomura Y. Induced morphisms for Lambek invariants of commutative squares. Manuscr. Math., 1971, vol. 4, iss. 3, pp. 263-275. DOI: https://doi.org/10.1007/BF01190280

8. Eckmann B., Hilton P. J. Exact couples in an abelian category. J. Algebra, 1966, vol. 3, pp. 38-87. DOI: https://doi.org/10.1016/0021-8693(66)90019-6

9. Kuz'minov V. I., Cherevikin A. Yu. Semiabelian categories. Siberian Math. J., 1972, vol. 13, no. 6, pp. 895-902. DOI: https://doi.org/10.1007/BF00971865

10. Yakovlev A. V. Homological algebra in pre-Abelian categories. J. Math. Sci., 1982, vol. 19, iss. 1, pp. 1060-1067. DOI: https://doi.org/10.1007/BF01476122

11. Rump W. Almost abelian categories. Cah. Topol. Géom. Différ. Catég., 2001, vol. 42, no. 3, pp. 163-225. 
12. Kopylov Ya. A., Wegner S.-A. On the notion of a semi-abelian category in the sense of Palamodov. Appl. Categor. Struct., 2012, vol. 20, pp. 531-541. DOI: https://doi.org/10.1007/s10485-011-9249-0

13. Schneiders J.-P. Quasi-abelian categories and sheaves. Mémoires de la Société Mathématique de France, Ser. 2, 1999, no. 76, 144 p. DOI: https://doi.org/10.24033/msmf.389

14. Bonet J., Dierolf S. The pullback for bornological and ultrabornological spaces. Note Mat., 2006, vol. 25, no. 1, pp. 63-67. DOI: https://doi.org/10.1285/i15900932v25n1p63

15. Rump W. A counterexample to Raikov's conjecture. Bull. Lond. Math. Soc., 2008, vol. 40, iss. 6, pp. 985-994. DOI: https://doi.org/10.1112/blms/bdn080

16. Rump W. Analysis of a problem of Raikov with applications to barreled and bornological spaces. J. of Pure Appl. Algebra, 2011, vol. 215, iss. 1, pp. 44-52. DOI: https://doi.org/10.1016/j.jpaa.2010.02.031

17. Wengenroth J. The Raikov conjecture fails for simple analytical reasons. J. Pure Appl. Algebra, 2012, vol. 216, iss. 7, pp. 1700-1703. DOI: https://doi.org/10.1016/j.jpaa.2012.01.007

18. Kelly G. M. Monomorphisms, epimorphisms, and pull-backs. J. Austral. Math. Soc., 1969, vol. 9, pp. 124-142. DOI: https://doi.org/10.1017/S1446788700005693

19. Gelfand I. M., Manin Yu. I. Methods of Homological Algebra. Springer Monographs in Mathematics. Berlin, Springer-Verlag, 2003. 372 p.

20. Kopylov Ya. A., Kuz'minov V. I. Exactness of the cohomology sequence corresponding to a short exact sequence of complexes in a semiabelian category. Siberian Adv. Math., 2003, vol. 13, no. 3, pp. 72-80.

21. Kopylov Ya. A. Homology in P-semi-abelian categories. Sci. Ser. A Math. Sci. (N.S.), 2009, vol. 17, pp. 105-114.

22. Kopylov Ya. A. On the homology sequence in a $P$-semi-abelian category. Sib. Elektron. Mat. Izv., 2012, vol. 9, pp. 190-200.

23. Makarov B. M. Some pathological properties of inductive limits of $B$-spaces. Uspekhi Mat. Nauk, 1963, vol. 18, iss. 3 (111), pp. 171-178 (in Russian).

Cite this article as:

Kopylov Ya. A. On Some Diagram Assertions in Preabelian and P-Semi-Abelian Categories. Izv. Saratov Univ. (N. S.), Ser. Math. Mech. Inform., 2020, vol. 20, iss. 4, pp. 434-443. DOI: https://doi.org/10.18500/1816-9791-2020-20-4-434-443

УДК 512.66:517.982.2

\section{О некоторых диаграммных утверждениях в предабелевых и $Р$-полуабелевых категориях}

\section{Я. А. Копылов}

Копылов Ярослав Анатольевич, кандидат фризико-математических наук, Институт математики им. С. Л. Соболева СО РАН, Россия, 630090, г. Новосибирск, просп. Ак. Коптюга, д. 4, yakop@math.nsc.ru

Как известно, многие важные аддитивные категории фрункционального анализа и алгебры неабелевы. Многие классические диаграммные утверждения, справедливые в абелевых категориях, оказываются неверны в более общих аддитивных категориях без дополнительных предположений о свойствах морфризмов рассматриваемых диаграмм. Это, в частности, относится к так называемой лемме о змее, или Ker-Coker-последовательности. 
В статье получена теорема о диаграмме, обобщающей классическую ситуацию леммы о змее в контексте категорий, полуабелевых в смысле Паламодова. Известно также, что уже в Р-полуабелевых категориях не все ядра (соответственно, коядра) полустабильны, т. е. стабильны относительно универсальных (соответственно, коуниверсальных) квадратов. Мы доказываем предложение, показывающее, как неполустабильные ядра и коядра могут возникнуть в общих предабелевых категориях.

Ключевые слова: Р-полуабелева категория, строгий морфизм, полустабильные ядра и коядра, лемма о змее, Ker-последовательность, Coker-последовательность.

Поступила в редакцию: 15.12.2019 / Принята: 23.03.2020 / Опубликована: 30.11.2020

Статья опубликована на условиях лицензии Creative Commons Attribution License (CC-BY 4.0)

Благодарности. Автор благодарит рецензента за ценные замечания, которые помогли существенно улучшить работу. Работа выполнена в рамках государственного задания ИМ СО РАН (проект № 0314-2019-0006).

\section{Образец для цитирования:}

Kopylov Ya. A. On Some Diagram Assertions in Preabelian and P-Semi-Abelian Categories [Kопьлов Я. A. О некоторых диаграммных утверждениях в предабелевых и Р-полуабелевых категориях] // Изв. Сарат. ун-та. Нов. сер. Сер. Математика. Механика. Информатика. 2020. T. 20, вып. 4. С. 434-443. DOI: https://doi.org/10.18500/1816-9791-2020-20-4-434-443 